\title{
Pevonedistat, a Nedd8-activating enzyme inhibitor, sensitizes neoplastic B-cells to death receptor-mediated apoptosis
}

\author{
Cody Paiva ${ }^{1, *}$, J. Claire Godbersen ${ }^{2, *}$, Taylor Rowland ${ }^{1}$, Olga V. Danilova ${ }^{3}$, \\ Christopher Danes ${ }^{4}$, Allison Berger ${ }^{4}$, Alexey V. Danilov ${ }^{1}$ \\ ${ }^{1}$ Knight Cancer Institute, Oregon Health and Science University, Portland, OR, USA \\ ${ }^{2}$ Geisel School of Medicine at Dartmouth, Department of Medicine, Hanover, NH, USA \\ ${ }^{3}$ VA Portland Healthcare System, Department of Pathology and Laboratory Medicine, Portland, OR, USA \\ ${ }^{4}$ Millennium Pharmaceuticals, Inc., a wholly owned subsidiary of Takeda Pharmaceutical Company Ltd., Cambridge, MA, USA \\ *These authors have contributed equally to this work
}

Correspondence to: Alexey V. Danilov, email: danilov@ohsu.edu

Keywords: lymphoma, neddylation, CLL, TRAIL

Published: February 03, 2017

\section{ABSTRACT}

\begin{abstract}
While death receptor ligands (Fas and TRAIL) kill chemoresistant tumor cell lines, related therapies have limited clinical efficacy as single agents. Death receptor signaling is modulated by nuclear factor-KB (NFKB), a family of transcription factors which are constitutively active in B-cell malignancies. We and others have shown that pevonedistat, an investigational inhibitor of the NEDD8-activating enzyme, abrogates NFKB activity in B-cell neoplasia. Here we demonstrate that diffuse large B-cell lymphoma, particularly activated B-cell type, and primary chronic lymphocytic leukemia cells are re-sensitized to extrinsic apoptosis by pevonedistat. Pevonedistat enhanced caspase-8 processing following death receptor ligation, and downmodulated cFLIP, a NFKB-regulated protease-deficient caspase homolog. However, treatment with pevonedistat did not modulate death-inducing signaling complex in neoplastic B-cells, suggesting that they were sensitized to death ligands through the mitochondrial pathway. Our data provide rationale for further development of pharmacologic agents including pevonedistat in strategies which enhance death receptor signaling in lymphoid malignancies.
\end{abstract}

\section{INTRODUCTION}

Apoptotic pathways are dependent on activation of initiator and executioner caspases (caspase-2/8/9/10 and $3 / 6 / 7$, respectively). Caspase activation can be induced at the plasma membrane (extrinsic apoptosis) or at the mitochondria (intrinsic apoptosis). Engagement of death receptors belonging to the tumor-necrosis factor (TNF) receptor gene superfamily, e.g. TRAIL-R1/2 (TNFrelated apoptosis-inducing ligand receptors; DR4/5) and Fas (CD95) by their respective cognate 'death ligands' TRAIL (Apo2L) and FasL (Apo1L), kills multiple tumor cell lines independent of their chemosensitivity or TP53 mutational status [1-5]. While Fas is highly toxic against hepatocytes and can induce fulminant liver injuries [6], TRAIL lacks toxicity in animal models, thus holding promise in oncology therapeutics. Whereas a number of preparations of TRAIL and its derivatives were safe in clinical trials, single agent efficacy data is disappointing, necessitating the development of novel combination approaches [4].

Among the factors which contribute to resistance to death ligands, the nuclear factor- $\mathrm{\kappa B}(\mathrm{NF} \kappa \mathrm{B})$-driven upregulation of the anti-apoptotic genes in response to death receptor ligation was shown to result in a diminished cellular susceptibility to extrinsic apoptosis across several tumor types [7-9]. The NFKB transcription factors modulate cell survival during stress and immune response [10]. Their anti-apoptotic function is fulfilled in part via regulation of the inhibitor of apoptosis (IAP) and Bcl-2 family members. Recent reports added controversy to the role of $\mathrm{NF \kappa B}$ in death receptor signaling, where individual $\mathrm{NF \kappa B}$ subunits were shown to play conflicting roles [11]. For example, the predominantly pro-survival activity of the RelA (p65) may be counterbalanced by pro-apoptotic effect of c-Rel. 
NFкB pathway deregulation contributes to oncogenesis in B-cell malignancies and is detected in both aggressive (diffuse large B-cell lymphoma [DLBCL]) and indolent (chronic lymphocytic leukemia/ small lymphocytic lymphoma [CLL]) non-Hodgkin lymphoma (NHL) subtypes [12, 13]. Gene expression profiling categorizes DLBCL based on cell-of-origin, where $\mathrm{NF} \kappa \mathrm{B}$ activation is the key feature of the less curable activated B-cell-like (ABC)-DLBCL [14]. However NFאB aberrations are also found in germinal center-like (GC)-DLBCL [12]. We and others have established that pevonedistat (MLN4924, TAK-924), an investigational inhibitor of the NEDD8-activating enzyme (NAE), successfully abrogates NFאB pathway activity in B-cell malignancies [15-17]. Interaction between NAE and NEDD8, a ubiquitin-like modifier, ultimately leads to activation of Cullin-RING ligases (CRL), followed by ubiquitination and degradation of their substrate proteins. Pevonedistat forms a covalent adduct with NEDD8, thereby disrupting this interaction, and leading to extended half-life of CRL substrates, including inhibitor of $\mathrm{NF} \kappa \mathrm{B}(\mathrm{I} \kappa \mathrm{B})[15,18]$. Recent clinical data shows that pevonedistat has a favorable adverse event profile in patients with hematologic malignancies [19, 20].

Given the pathogenic role of $\mathrm{NF} \kappa \mathrm{B}$ in lymphoma, and its role in resistance to death ligands, we studied whether NAE inhibition sensitizes neoplastic B-cells to extrinsic apoptosis.

\section{RESULTS}

\section{NAE inhibition sensitizes neoplastic B-cells to extrinsic apoptosis}

We studied expression of TRAIL-R and Fas (CD95) in a panel of DLBCL cell lines. TRAIL-R1 (DR4) was expressed in all tested DLBCL cell lines, while TRAIL-R2 (DR5) was highly expressed in ABC-DLBCL and in 3/7 tested GC-DLBCL cell lines (Figure 1). By contrast, Fas was expressed at low levels, while Fas-associated death domain (FADD) adaptor protein was detectable in all DLBCL cell lines (Figure 1A). Cell surface expression of TRAIL-R1/2 and Fas was confirmed by flow cytometry (Figure 1B). "Decoy" receptors TRAIL-R3/4, which are unable to transmit apoptotic signals and thus may foster resistance to TRAIL-mediated apoptosis [21], were expressed at low levels (Figure 1B).

Despite this, DLBCL cells were resistant to both TRAIL and Fas ligand used in concentrations sufficient to induce killing of Jurkat cells (up to $10 \mathrm{ng} / \mathrm{mL}$, data not shown and [22, 23]; Figure 2 and Supplementary Figure 1). Exposure to high concentration of ligands $(100 \mathrm{ng} / \mathrm{mL})$ led to minimal cell apoptosis (Figure 2).

Meanwhile, NAE inhibitor pevonedistat sensitized DLBCL cells to death receptor agonists. Pevonedistat alone used at a clinically achievable concentration of 0.5 $\mu \mathrm{M}$ [19], induced $<20 \%$ apoptosis, in both $\mathrm{ABC}$ - and GCDLBCL cells (Figure 2, gray bars on the left). However, ABC-DLBCL cells were sensitized to death receptor signaling (Figure 2 and Supplementary Figure 1). Of note, $68.5 \pm 5.2 \%$ OCI-LY3 cells underwent apoptosis following treatment with pevonedistat combination with $10 \mathrm{ng} / \mathrm{mL}$ Fas ligand, versus $25.2 \pm 4.7 \%$ with Fas ligand alone. Pan-caspase inhibition rendered partial protection from apoptosis (Supplementary Figure 2). By contrast, GC-DLBCL cells exhibited modest, if any, sensitization (Figure 2).

Thus, DLBCL cells were resistant to extrinsic apoptosis irrespective of the cell of origin, while pevonedistat sensitized DLBCL cells (predominantly $\mathrm{ABC}$-like) to death receptor agonists.

\section{Targeting NAE enhances extrinsic apoptosis in CD40-stimulated primary CLL cells}

We then studied whether our findings could be replicated in primary neoplastic B-cells. Our group has previously demonstrated that targeting NAE potently inhibits NFאB in primary CLL cells under the conditions mimicking the lymph node microenvironment [15, 16]. Here, as previously, CLL cells were cultured in the presence of CD40L-expressing stroma, leading to activation of the canonical and non-canonical NFKB pathways, and resistance to spontaneous and druginduced apoptosis, as previously shown by us and others $[15,24]$. Death receptors and their ligands, notably Fas, FasL and TRAIL, are regulated by NFאB [25-27]. While unstimulated CLL cells derived from peripheral blood had low death receptor expression, CD40L stimulation upregulated Fas and TRAIL-R2 (but not TRAIL-R1) as well as "decoy" receptors TRAIL-R3/4 in CLL cells (Figure 3A-3B). Despite this, treatment with TRAIL or FasL alone did not kill CLL cells (Figure 3C). Meanwhile, NAE inhibition did not affect death receptor expression (Figure 3A), but resulted in sensitization of the CD40stimulated CLL cells to TRAIL (Figure 3C).

In agreement with our earlier findings [15], CD40Lstimulated CLL cells were sensitized to pevonedistat compared to cells co-cultured with stroma control (Figure 3D). Given that autologous cytotoxic T-lymphocytes, NK cells and monocytes present in co-cultures could secrete endogenous death receptor ligands, we inquired whether enhanced death receptor expression could explain this finding. However, neutralizing antibodies against TNF $\alpha$, FasL (CD178) and TRAIL (CD263), alone or in combination, did not render protection from pevonedistatinduced apoptosis (Figure 3E).

Finally, engagement of CD40 promotes recruitment of adapter proteins known as TNF receptor-associated factors (TRAFs) to the receptor cytoplasmic domains [28, 29]. TRAF signaling relies on a series of ubiquitylation events which could potentially be modulated by NAE 
inhibition. CD40 directly associates with TRAF2, 3 and 6, where TRAF2 activates the cIAP to ubiquitylate TRAF3, promoting its degradation. While CD40L stimulation induced expression of NFKB-regulated cIAPs [30, 31] in CLL cells, NAE inhibition did not alter their expression, suggesting that CD40/TRAF signaling is unperturbed when NAE is inhibited (Figure 3D).

Thus, pevonedistat sensitized primary CLL cells to exogenous death receptor ligands.

\section{Pevonedistat enhances caspase-8 activation in neoplastic B-cells}

We next sought to ascertain if targeting NAE facilitated extrinsic apoptosis in neoplastic B-cells. First, we determined whether pevonedistat promoted caspase- 8 activation, a key effector caspase involved in death receptor signaling. OCI-LY3 cells which underwent short-term stimulation with TRAIL (but not

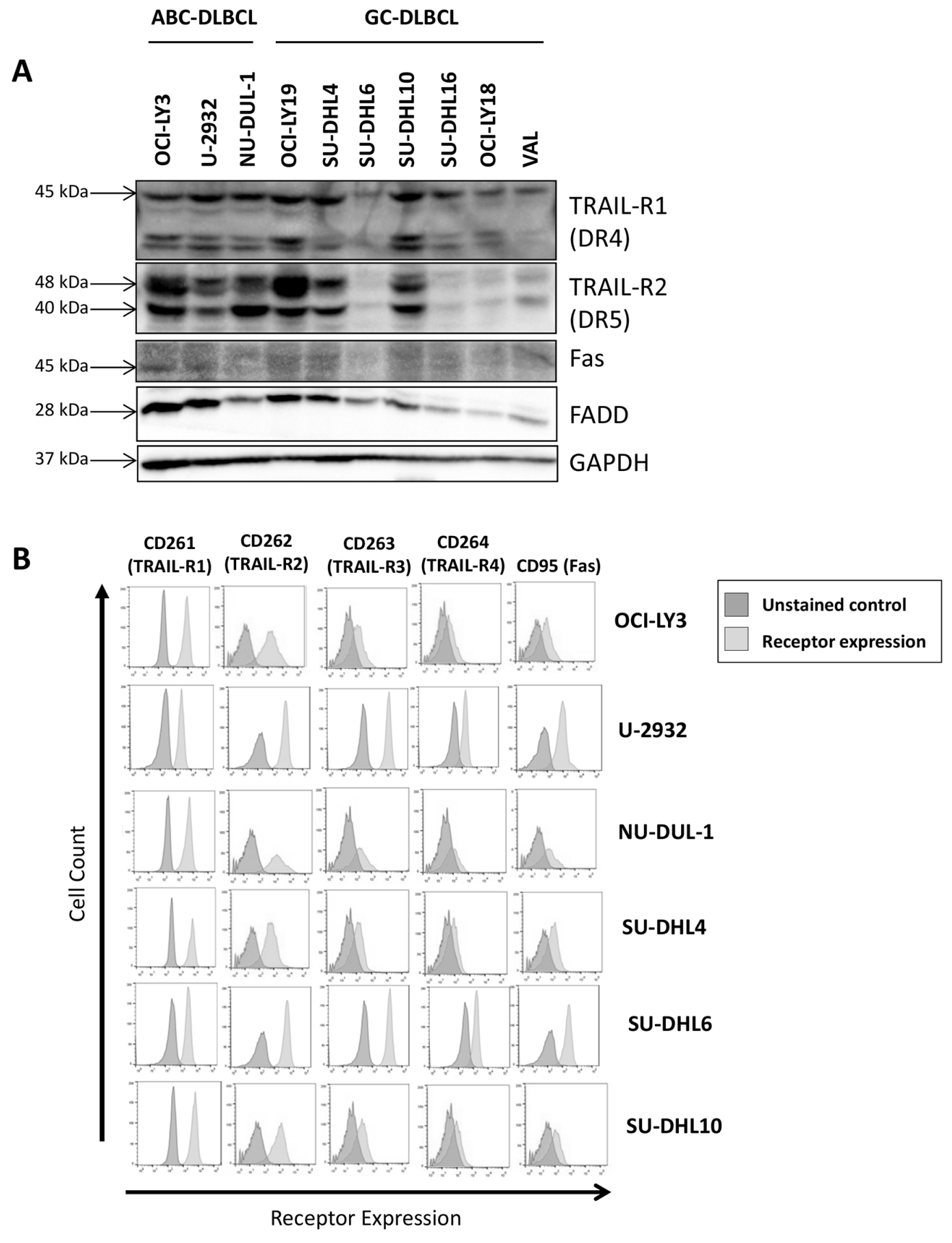

Figure 1: Death receptor expression in DLBCL cell lines was determined in whole-cell protein lysates by immunoblotting A. and by flow cytometry $\mathbf{B}$. 
Fas ligand) demonstrated rapid caspase- 8 cleavage, loss of unprocessed Bid and apoptosis (as evidenced by enhanced PARP cleavage; Figure 4A). Conditioning of either OCILY3 or primary CLL cells with pevonedistat, but not Bcl-2 inhibitor venetoclax, enhanced TRAIL-mediated caspase- 8 activation (Figure 4A-4B).
TNFR-mediated apoptosis requires assembly of a functional death-inducing signaling complex (DISC), which is composed of several signaling adaptors (e.g., FADD) and leads to caspase- 8 and Bid processing. c-FLIP, a protease-deficient caspase homolog, is concurrently recruited to the DISC where it inhibits caspase- 8 processing. Thus, the balance between c-FLIP
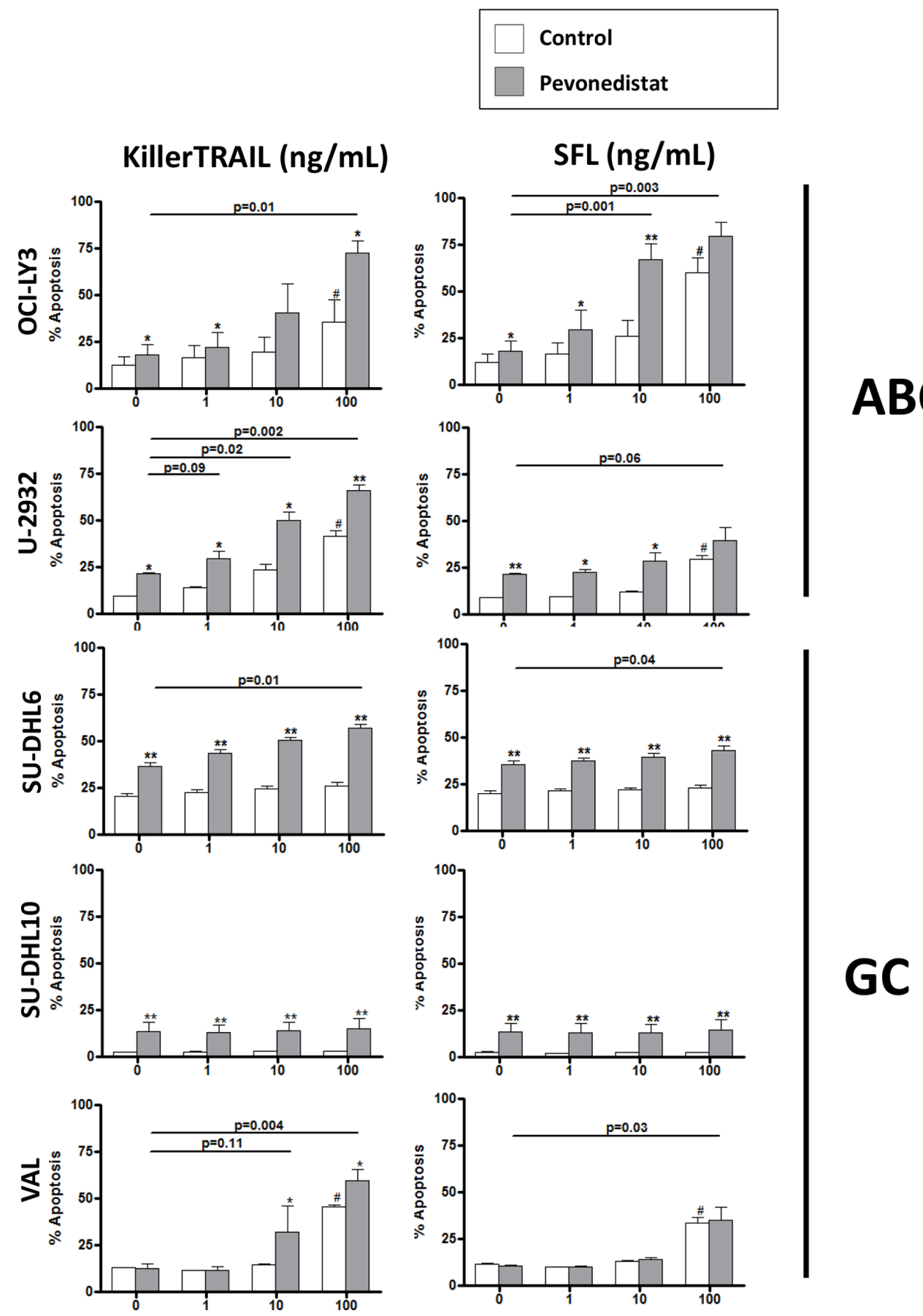

Figure 2: Pevonedistat sensitizes DLBCL cells to death receptor agonists. Cell lines were incubated with the indicated concentrations of KillerTrail and SuperFasLigand (SFL) and $0.5 \mu \mathrm{M}$ pevonedistat (or vehicle control) for 24 hours. Apoptosis was determined by Annexin V staining. Data are the mean \pm SE of three independent experiments. ${ }^{*}-\mathrm{p}<0.05$ compared to untreated control; $*-p<0.05$ and $* *-p<0.01$ when comparing death receptor agonist/pevonedistat combination with the agonist alone. 
and caspase- 8 levels determines the outcome of death receptor signaling [32-34]. c-FLIP is a bona fide NFkB transcriptional target, and targeting NAE reduces c-FLIP mRNA and protein in CD40-stimulated CLL cells (Figure $3 \mathrm{D}$ and [15]). By contrast, c-FLIP protein levels were modestly reduced in pevonedistat-treated OCI-LY3 cells (Figure 4C).

We supposed that NAE inhibition could lead to decreased participation of c-FLIP in the DISC, thereby promoting caspase- 8 processing. Immune-precipitation experiments employing Apo-1L antibody (FasL) demonstrated DISC assembly and caspase- 8 activation, as manifested by generation of its active forms ( $\mathrm{p} 41 / 43)$ [35], in OCI-LY3 cells following Fas engagement (Figure 4D). Still, pevonedistat did not modulate recruitment of c-FLIP or caspase- 8 to the DISC, at least in response to FasL stimulation (Figure 4D). We could not analyze DISC in response to TRAIL because of the technical difficulties of DISC isolation using TRAIL antibody.

Our group and others have shown that NAE inhibition induces apoptosis of the neoplastic B-cells through the rebalancing of the Bcl-2 family members towards the pro-apoptotic BH3-only proteins Bim and Noxa; Cdt1 accumulation, DNA damage and checkpoint

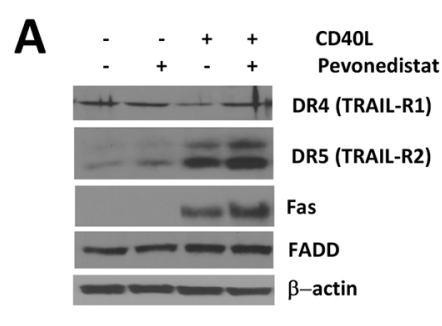

B
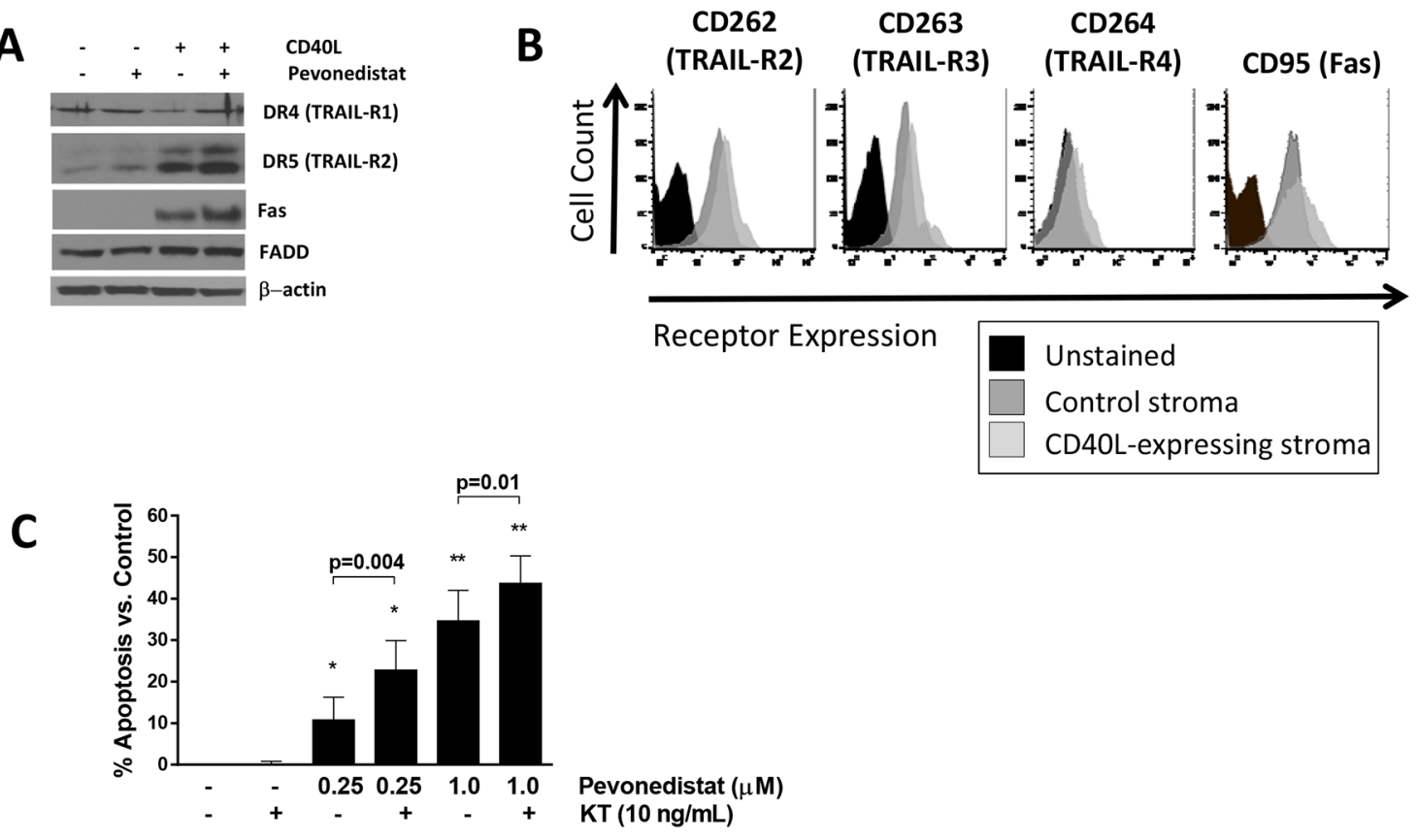

D
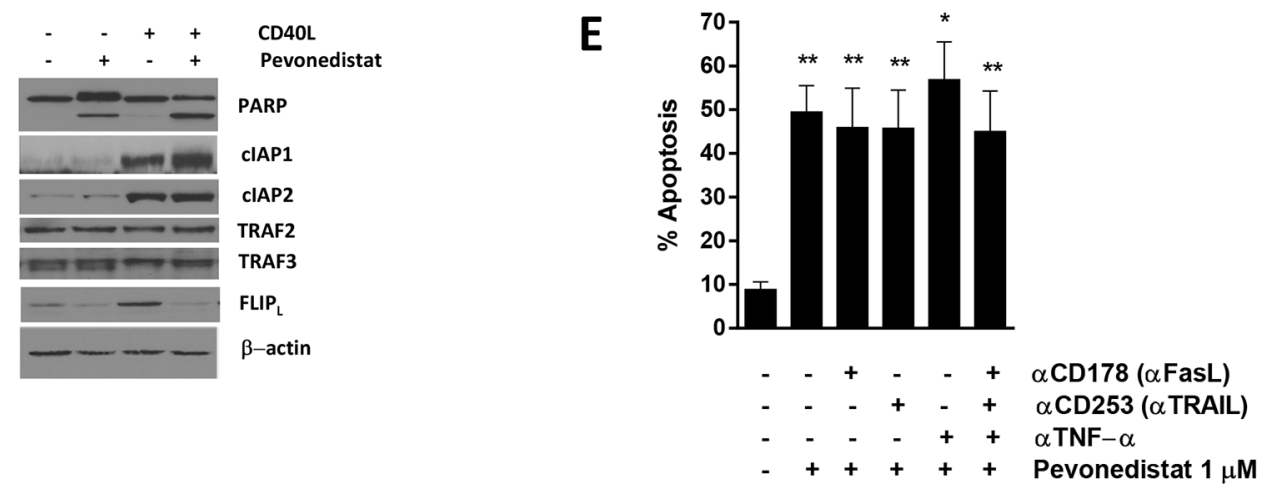

Figure 3: Pevonedistat sensitizes CLL cells to death receptor agonists. A and $\mathbf{B}$. CLL cells were co-cultured with CD40Lexpressing or control stroma for 24 hours and analyzed by flow cytometry (B), followed by incubation with $1 \mu \mathrm{M}$ pevonedistat or vehicle control for 24 hours. Cells were lysed and subjected to immunoblotting. A representative blot from 4 independent experiments is shown. C. CLL cells $(\mathrm{N}=6)$ were co-cultured with CD40L-expressing for 24 hours and subsequently incubated with the indicated drugs for 24 hours. Apoptosis was determined by Annexin V and 7-AAD staining within the CD19+ subset of cells. Data are mean \pm SE. Values were normalized to untreated control. D. CLL cells were treated as in (A). E. CLL cells $(\mathrm{N}=4)$ were co-cultured with CD40L-expressing for 24 hours and then treated with $1 \mu \mathrm{M}$ pevonedistat, the indicated antibodies $(0.5 \mu \mathrm{g})$, or the combination for 24 hours. Apoptosis was determined by Annexin $\mathrm{V}$ and 7-AAD staining within the CD19 ${ }^{+}$subset of cells. Data are mean \pm SE. ${ }^{*}-\mathrm{p}<0.05, * *-\mathrm{p}<0.01$ compared with untreated control. 
activation $[15,17,36]$. Here we observed caspase-8 processing in OCI-LY3 cells after 1 hour incubation with pevonedistat, albeit to a lesser extent than in response to death ligands (Figure 4E). This suggested that pevonedistat could promote casepase- 8 cleavage by enhancing intrinsic (mitochondrial) apoptosis.
Consistent with that, Bcl-2 inhibitor venetoclax and SMAC-mimetic LCL161 also sensitized OCI-LY3 cells to FasL (Figure 4F).

Thus, pevonedistat augmented extrinsic apoptosis in neoplastic B-cells via enhanced caspase- 8 activation.

\section{A}

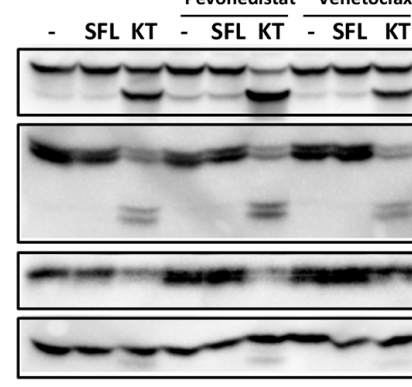

C

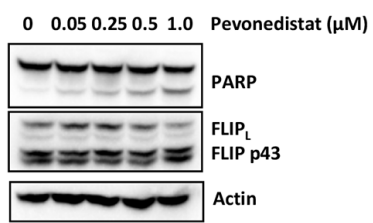

B

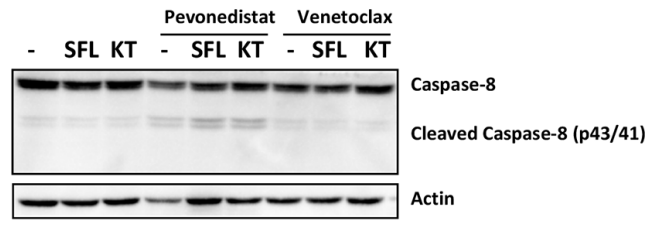

Cleaved Caspase-8 (p43/41)

Bid Actin

D

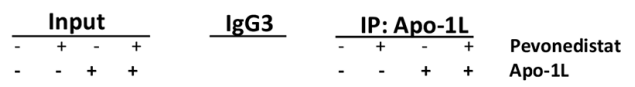

$\mathbf{E}$
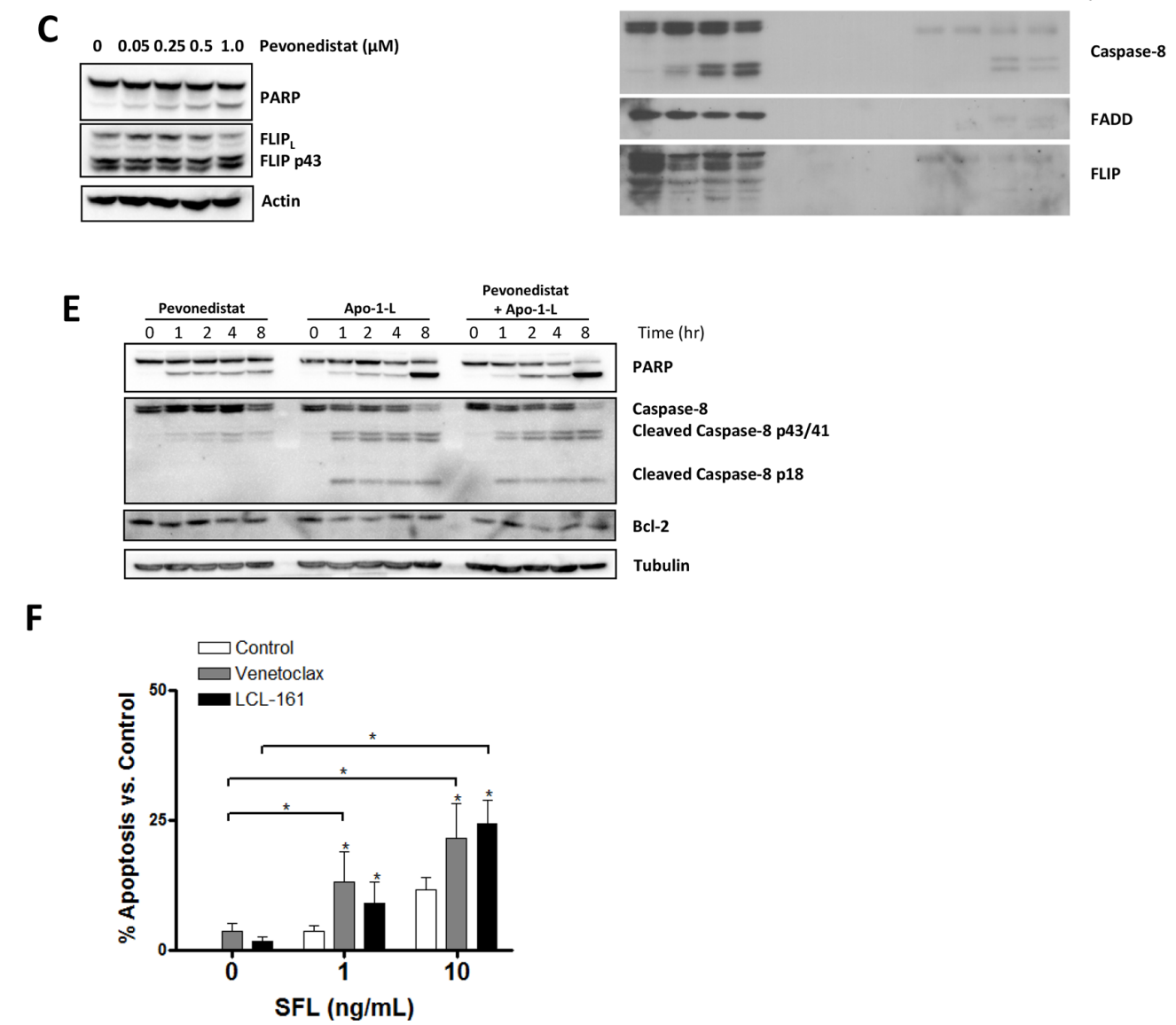

Figure 4: NAE inhibition enhances caspase-8 activation in neoplastic B-cells. A. OCI-LY3 cells were incubated with $0.5 \mu \mathrm{M}$ pevonedistat, venetoclax or vehicle control for 24 hours, followed by treatment with $10 \mathrm{ng} / \mathrm{mL} \mathrm{KT}$ or $10 \mathrm{ng} / \mathrm{mL} \mathrm{SFL}$ for 1 hour. Cells were lysed and subjected to immunoblotting. B. CLL cells were co-cultured with CD40L-expressing stroma for 24 hours, followed by incubation with $0.5 \mu \mathrm{M}$ pevonedistat, venetoclax or vehicle control for 24 hours, and then stimulated with $10 \mathrm{ng} / \mathrm{mL} \mathrm{KT}$ or SFL for 1 hour, lysed and subjected to immunoblotting. C. OCI-LY3 cells were incubated with the indicated concentrations of pevonedistat for 24 hours, lysed and subjected to immunoblotting. Representative blots of at least 3 independent experiments are shown. D. OCI-LY3 cells were incubated with $0.5 \mu \mathrm{M}$ pevonedistat or vehicle control for 24 hours, and then stimulated or not with $100 \mathrm{ng} / \mathrm{mL}$ Apo- $1 \mathrm{~L}$. Cells were lysed and subjected to immunoprecipitation using Apo-1L antibody as described in the methods. E. OCI-LY3 cells were treated with $0.5 \mu \mathrm{M}$ pevonedistat, $100 \mathrm{ng} /$ $\mathrm{mL}$ Apo-1L, or drug combination. Cells were lysed at the indicated timepoints and subjected to immunoblotting. (E) OCI-LY3 cells were incubated with $1 \mu \mathrm{M}$ venetoclax, $1 \mu \mathrm{M}$ LCL-161, and the indicated concentrations of SFL for 24 hours. Apoptosis was assessed by Annexin V staining and values normalized to untreated control. * - $p<0.05$ compared to untreated control. 


\section{DISCUSSION}

Survival of the neoplastic B-cells depends on the misbalance between the pro- and anti-apoptotic molecules $[37,38]$. Venetoclax, a novel BH3-mimetic which targets the pro-survival protein Bcl-2, has been recently approved for therapy of patients with relapsed/refractory CLL with del (17p), where it is highly efficacious [39]. By contrast, responses to venetoclax are short-lived in NHL, including DLBCL [40]. Thus, novel strategies to re-activate apoptosis are necessary. At this time, multiple clinical trials explore venetoclax in combination with inhibitors of B-cell receptor-associated kinases (e.g., ibrutinib) and $\mathrm{CD} 20$-targeting agents (e.g., rituximab) in NHL [41]. Here, extending our earlier findings on feasibility of targeting NFKB in neoplastic B-cells, we propose a potential therapeutic approach which employs NAE inhibitor pevonedistat to facilitate extrinsic apoptosis in lymphoma.

We demonstrate that despite high surface expression of death receptors TRAIL-R1/2 and Fas, ABC-and GC-DLBCL cells exhibit resistance to death ligands. Given the involvement of the NFKB pathway in regulation of death receptor signaling and its antiapoptotic effect in this setting [7-9], we investigated strategies to promote extrinsic apoptosis through targeting NFkB. Pevonedistat, an investigational NAE inhibitor, abrogates NFKB activity in DLBCL [17]. Indeed, pevonedistat sensitized DLBCL cell lines to death ligands. While constitutive activation of the NFKB pathway has been detected across the DLBCL subtypes, it is indispensable for proliferation and survival of ABC-like DLBCL [12, 42]. Targeting NAE potently inhibits NFKB in ABC-DLBCL; by contrast, GC-like undergo DNA re-replication and damage and checkpoint activation when NAE is inhibited [17]. Consistent with this, ABC-DLBCL showed enhanced apoptosis in response to combined treatment with pevonedistat and death ligands, compared with GC-DLBCL.

Unstimulated primary CLL cells sourced from peripheral blood are resistant to extrinsic apoptosis [34, 43]. In agreement with earlier findings, we demonstrate that $\mathrm{CD} 40 \mathrm{~L}$ stimulation, which partially mimics the lymph node microenvironment, induces death receptor expression [44]. This renders an opportunity to co-opt extrinsic apoptotic pathways in the CLL microenvironment niche in vivo. However, like DLBCL cells, primary CLL cells co-cultured with CD40Lexpressing stroma in vitro were resistant to death ligands. Our data appears to be in agreement with results by others where withdrawal of soluble CD40L was necessary to achieve complete activation of FasL/Fas system in CLL [44]. Importantly, CD40-stimulated CLL cells were sensitized to death receptor signaling by pevonedistat, which we have shown to be a potent NFKB inhibitor in those cells $[15,16]$. Interestingly, neutralization of endogenous death ligands, presumably present in CLLstromal cell co-cultures, did not protect CLL cells from NAE inhibition, suggesting that the pro-apoptotic activity of pevonedistat did not depend on receptor apoptotic pathways.

It is notable that compared to ABC-DLBCL, CLL cells are less efficiently sensitized to extrinsic apoptosis by pevonedistat. Indeed, several mechanisms which prevent caspase- 8 activation and thereby contribute to the 'dysfunctional' death receptor signaling have been identified in CLL. Resistance of peripheral blood CLL cells to extrinsic apoptosis may be explained in part by low death receptor expression, accompanied by suboptimal DISC formation in response to TRAIL [34]. High c-FLIP ${ }_{L}$ to caspase-8 ratio, accompanied by inefficient processing of the latter, may further contribute to resistance [34]. Lyn, a Src family kinase constitutively activated and involved in B-cell receptor signal transmission in CLL cells, has also been implicated in resistance to extrinsic apoptosis: Lyn induces phosphorylation of procaspase- 8 , thereby promoting the formation of enzymatically inactive homodimer [45]. It is possible that some of the above mechanisms may underlie DLBCL cell resistance to death receptor agonists as single agents.

Our data suggests that NFKB contributes to resistance to death receptor signaling in neoplastic B-cells, which can be partially reversed by NAE inhibition. c-FLIP, a bona fide NFKB transcriptional target, participates in DISC assembly and counterbalances caspase- 8 activation $[32,33]$. Interestingly, NAE inhibition had differential effects on c-FLIP expression in DLBCL cells and primary CLL cells, where its downregulation was modest in the former and pronounced in the latter. Treatment with pevonedistat did not seem to enhance DISC formation or c-FLIP participation in the DISC in response to FasL in OCI-LY3 cells. However, it is important to note that NAE inhibition enhanced caspase- 8 processing in response to TRAIL more efficiently, and thus enhanced DISC formation cannot be fully excluded.

CD40L stimulation of the neoplastic B-cells derived from the lymph nodes of patients with follicular lymphoma induced $\mathrm{NF} \kappa \mathrm{B}$, accompanied by rapid upregulation of Bcl$\mathrm{xL}$ and c-FLIP, leading to TRAIL resistance [46]. CD40L stimulation induces anti-apoptotic Bcl-2 family members in primary CLL cells, including Bcl-X, Bcl-2 and Mcl-1 $[15,47,48]$. NAE inhibition leads to rebalancing of the Bcl-2 family proteins in favor of apoptosis, accompanied by a downregulation of the pro-survival Bcl-2 family members and an increase in pro-apoptotic BH3-only proteins Bim and Noxa $[15,17]$. We found that treatment with pevonedistat alone was able to initiate caspase- 8 cleavage. Thus, it is possible that NAE inhibition enhances caspase-8 processing through a feedback loop mediated by activation of caspase- 3 via intrinsic (mitochondrial) pathways [49]. 
Other strategies to enhance sensitivity of the neoplastic B-cells to extrinsic apoptosis have been reported. Histone deacetylase inhibitors enhance FADD recruitment to TRAIL-R1 and thereby sensitize CLL cells to TRAIL-apoptosis [50]. The proteolytic activity of caspase- 3 can be inhibited by X-linked inhibitor of apoptosis (XIAP) and a novel class of drugs called second mitochondria-derived activator of caspase (SMAC)mimetics directly bind and antagonize XIAP, sensitizing CLL cells to TRAIL $[51,52]$. Indeed, we found that both Bcl-2 inhibitor venetoclax, and SMAC-mimetic LCL161 sensitized OCI-LY3 cells to FasL, suggesting that those represent viable potential combination strategies in lymphoma.

Pevonedistat has previously been shown to synergize with TNF $\alpha$ to induce apoptosis in monocytes, dendritic cells and hepatocytes via induction of both apoptotic and necroptotic pathways [53, 54]. This may raise concerns regarding synergistic toxicity. However, in a recently reported Phase I study of pevonedistat in NHL, grade $\geq 3$ transaminase increases were observed in less than $5 \%$ of patients (2/44) [19]. Nevertheless, we would advocate for a careful dose escalation schema and a period of observation following administration of TRAIL agonists in combination with pevonedistat in a clinical trial setting.

Additional investigations are needed to better define how to best use pevonedistat in patients with B-cell malignancies. We have previously reported that pevonedistat enhances the pro-apoptotic effect of the DNA-damaging agent bendamustine in CLL cells [36]. However, in the current era, 'targeted' therapies are receiving widespread use in lymphoid malignancies, and are notable for their favorable toxicity profile compared with standard chemotherapy drugs [55]. Thus, combination strategies which involve inhibitors of B-cell receptor-associated kinases and pevonedistat, as previously reported by us [15], seem most attractive.

In summary, our data in primary neoplastic B-cells is consistent with findings by others that pevonedistat enhances the anti-tumor activity of TRAIL in solid tumor cell lines [56], thus suggesting that pevonedistat should be further investigated in therapy of CLL and NHL.

\section{MATERIALS AND METHODS}

\section{Primary CLL samples and stromal cell co-cultures}

Peripheral blood was obtained from patients with CLL at Dartmouth-Hitchcock Medical Center and Oregon Health and Science University following approval by the respective Institutional Review Board and written informed consent of patients. Of 25 patients who provided samples for this study, $80 \%$ of patients were previously untreated. $40 \%$ had $\operatorname{del}(13 q), 20 \%$ - trisomy $12,24 \%$ had no chromosomal abnormalities as detected by FISH; this information was not available in the remaining patients. Peripheral blood mononuclear cells (PMBCs) were isolated using standard Ficoll-Hypaque techniques (Amersham, Piscataway, NJ). Such CLL samples contained $\geq 90 \% \mathrm{CD}^{+} / \mathrm{CD} 19^{+}$cells, as determined by flow cytometry. CLL cells were cultured in RMPI-1640 supplemented with $15 \%$ fetal bovine serum, $100 \mathrm{U} / \mathrm{mL}$ penicillin, $100 \mu \mathrm{g} / \mathrm{mL}$ streptomycin, $2 \mathrm{mM}$ L-glutamine, $25 \mathrm{mM}$ HEPES, $100 \mu \mathrm{M}$ non-essential amino acids and $1 \mathrm{mM}$ sodium pyruvate (Lonza, Walkersville, MD). All experiments were performed with freshly isolated CLL cells.

The mouse fibroblast cell line (L cells) engineered to express CD40L (L4.5) was given to us by Dr. Sonia Neron (Université Laval, Quebec, Canada)[57]. Parental L cells were obtained from American Type Culture Collection (Manassas, VA). Both cell lines were maintained in DMEM with $10 \%$ FBS and penicillin-streptomycin. CLL cells were cultured under standardized conditions on stromal cells as previously described [15]. Briefly, stromal cells were seeded to achieve $80-100 \%$ confluence; on the following day, CLL cells were plated at a 50:1 ratio and incubated at $37^{\circ} \mathrm{C}$ in $5 \% \mathrm{CO}_{2}$. At harvest, CLL cells were gently washed off the stromal layer. When collected for protein analysis, CLL cells were transferred to a new plate and incubated for an additional 60 minutes to allow re-attachment of stromal cells and thus minimize contamination of CLL cells.

DLBCL cell lines Nu-DUL-1, SU-DHL4, SUDHL6, SU-DHL10 and SU-DHL16 were obtained from American Type Culture Collection (Manassas, VA); OCILY18, U-2932 and VAL - from DSMZ (Braunschweig, Germany); OCI-LY3 and OCI-LY19 cells were a kind gift from Dr. Andrew Evens (Tufts University).

\section{Cell viability testing and drugs}

Cell apoptosis was measured in duplicate as previously described using the ApoScreen Annexin V Apoptosis Kit [58]. Briefly, cells were resuspended in 150 $\mu \mathrm{L}$ of Annexin $\mathrm{V}$ binding buffer containing $1 \mu \mathrm{L}$ each of Annexin V-PE, 7-aminoactinomycin (7-AAD) and CD19FITC (Southern Biotech, Birmingham, AL), followed by flow cytometry on a MACSQuant (Miltenyi Biotec, San Diego, CA).

To measure cell proliferation, cells were plated in 96-well plates (3000/well in $100 \mu \mathrm{L}, 6$ wells per sample) with drugs and incubated for 48 hours at $37^{\circ} \mathrm{C}$ in $5 \% \mathrm{CO}_{2}$. After incubation, relative numbers of viable cells were measured using a tetrazolium-based colorimetric assay (CellTiter AQueous One Solution Cell Proliferation Assay; Promega, Madison, WI).

The following drugs and antibodies were used: pevonedistat (provided by Millennium Pharmaceuticals Inc., Cambridge, MA); venetoclax (ABT-199, Activ 
Biochem, Maplewood, NJ), both diluted in DMSO; Fas agonists Apo-1L and SuperFasLigand (SFL; Enzo Life Sciences, Framingdale, NY); KillerTRAIL (KT; Enzo Life Sciences), diluted in sterile water; pan-caspase inhibitor QVD-OPh (Sigma Aldrich, St. Louis, MO, diluted in DMSO); neutralizing antibodies against FasL (CD178), TRAIL (CD253) and TNF- $\alpha$ (BD Biosciences, San Diego, CA). Fluorochromeconjugated antibodies to CD95 (Fas), CD261 (DR4, TRAIL-R1), CD262 (DR5, TRAIL-R2), CD263 (TRAIL-R3) and CD264 (TRAIL-R4) were purchased from Miltenyi Biotec.

\section{Immunoblotting and immunoprecipitation (IP)}

Proteins were analyzed by immunoblotting as previously described [59]. Briefly, cells were lysed in RIPA buffer (20 mM Tris, $150 \mathrm{mM} \mathrm{NaCl,} \mathrm{1 \%} \mathrm{NP-40,}$ $1 \mathrm{mM}$ NaF, $1 \mathrm{mM}$ Sodium phosphate, $1 \mathrm{mM} \mathrm{NaVO}$, $1 \mathrm{mM}$ EDTA, $1 \mathrm{mM}$ EGTA, supplemented with protease inhibitor cocktail (Roche, Indianapolis, IN), phosphatase inhibitor cocktail 2 (Sigma-Aldrich) and $1 \mathrm{mM}$ phenylmethanesulfonyl fluoride (PMSF). The following antibodies were used: Bcl-2, Bid, cFLIP, cellular IAP (cIAP)-1, cIAP2, caspase-8, FADD, Fas, PARP and cleaved PARP, TRAF2, TRAF3, TRAIL-R2/ DR5 (Cell Signaling, Danvers, MA); TRAIL-R1/DR4 (Santa Cruz Biotechnology, Santa Cruz, CA), $\beta$-actin, $\gamma$-tubulin (Sigma-Aldrich), horseradish peroxidaseconjugated anti-mouse and anti-rabbit antibodies (Cell Signaling).

For DISC IP, OCI-LY3 cells were stimulated with Apo-1L for 1 hour and lysed in immunoprecipitation lysis buffer (150 mM NaCl, $30 \mathrm{mM}$ Tris, 10\% Glycerol, 1\% Triton-X-100) supplemented as above. Protein lysates were incubated with Apo-1L or isotype control antibody at $4^{\circ} \mathrm{C}$ overnight, and then with protein $\mathrm{A}$ agarose beads (Cell Signaling) at $4^{\circ} \mathrm{C}$ for 2 hours. Samples were reconstituted in loading dye, heated at $95^{\circ} \mathrm{C}$ for $5 \mathrm{~min}$ and analyzed by immunoblotting, alongside input controls (at $1: 10)$.

\section{Statistical analysis}

Results of individual experiments were analyzed using paired and unpaired Student's $t$-test, Fisher Exact test, non-parametric Mann-Whitney test and Spearman $r$. Statistical analyses were completed using the GraphPad Prism 6 software package (La Jolla, CA). All tests were two-sided, and data were considered to be statistically significant at $\mathrm{p}<0.05$.

\section{CONFLICTS OF INTEREST}

The authors declare no conflicts of interest.

\section{GRANT SUPPORT}

AVD is supported by the Lymphoma Research Foundation Clinical Investigator Career Development Award.

\section{REFERENCES}

1. Wilson NS, Dixit V, Ashkenazi A. Death receptor signal transducers: nodes of coordination in immune signaling networks. Nat Immunol. 2009; 10:348-355.

2. Walczak H, Miller RE, Ariail K, Gliniak B, Griffith TS, Kubin M, Chin W, Jones J, Woodward A, Le T, Smith C, Smolak P, Goodwin RG, et al. Tumoricidal activity of tumor necrosis factor-related apoptosis-inducing ligand in vivo. Nature medicine. 1999; 5:157-163.

3. Kastan M. On the TRAIL from p53 to apoptosis? Nat Genet. 1997; 17:130-131.

4. Micheau O, Shirley S, Dufour F. Death receptors as targets in cancer. Br J Pharmacol. 2013; 169:1723-1744.

5. Fulda S, Los M, Friesen C, Debatin KM. Chemosensitivity of solid tumor cells in vitro is related to activation of the CD95 system. Int J Cancer. 1998; 76:105-114.

6. Ogasawara J, Watanabe-Fukunaga R, Adachi M, Matsuzawa A, Kasugai T, Kitamura Y, Itoh N, Suda T, Nagata S. Lethal effect of the anti-Fas antibody in mice. Nature. 1993; 364:806-809.

7. Ravi R, Bedi GC, Engstrom LW, Zeng Q, Mookerjee B, Gelinas C, Fuchs EJ, Bedi A. Regulation of death receptor expression and TRAIL/Apo2L-induced apoptosis by NF-kappaB. Nature cell biology. 2001; 3:409-416.

8. Ehrhardt H, Fulda S, Schmid I, Hiscott J, Debatin KM, Jeremias I. TRAIL induced survival and proliferation in cancer cells resistant towards TRAIL-induced apoptosis mediated by NF-kappaB. Oncogene. 2003; 22:3842-3852.

9. Schram BR, Rothstein TL. NF-kappa B is required for surface Ig-induced Fas resistance in B cells. J Immunol. 2003; 170:3118-3124.

10. Baeuerle PA, Baltimore D. NF-kappa B: ten years after. Cell. 1996; 87:13-20.

11. Chen X, Kandasamy K, Srivastava RK. Differential roles of RelA (p65) and c-Rel subunits of nuclear factor kappa B in tumor necrosis factor-related apoptosis-inducing ligand signaling. Cancer research. 2003; 63:1059-1066.

12. Compagno M, Lim WK, Grunn A, Nandula SV, Brahmachary M, Shen Q, Bertoni F, Ponzoni M, Scandurra M, Califano A, Bhagat G, Chadburn A, Dalla-Favera R, et al. Mutations of multiple genes cause deregulation of NF-kappaB in diffuse large B-cell lymphoma. Nature. 2009; 459:717-721.

13. Herishanu Y, Perez-Galan P, Liu D, Biancotto A, Pittaluga S, Vire B, Gibellini F, Njuguna N, Lee E, Stennett L, Raghavachari N, Liu P, McCoy JP, et al. The lymph node microenvironment promotes B-cell receptor signaling, 
NF-kappaB activation, and tumor proliferation in chronic lymphocytic leukemia. Blood. 2011; 117:563-574.

14. Alizadeh AA, Eisen MB, Davis RE, Ma C, Lossos IS, Rosenwald A, Boldrick JC, Sabet H, Tran T, Yu X, Powell JI, Yang L, Marti GE, et al. Distinct types of diffuse large B-cell lymphoma identified by gene expression profiling. Nature. 2000; 403:503-511.

15. Godbersen JC, Humphries LA, Danilova OV, Kebbekus PE, Brown JR, Eastman A, Danilov AV. The Nedd8-activating enzyme inhibitor MLN4924 thwarts microenvironmentdriven NF-kappaB activation and induces apoptosis in chronic lymphocytic leukemia B cells. Clin Cancer Res. 2014; 20:1576-1589.

16. Godbersen JC, Paiva C, Danilova OV, Berger A, Brown JR, Danilov AV. Targeting neddylation effectively antagonizes nuclear factor-kappaB in chronic lymphocytic leukemia B-cells. Leukemia \& lymphoma. 2015; 56:1566-1569.

17. Milhollen MA, Traore T, Adams-Duffy J, Thomas MP, Berger AJ, Dang L, Dick LR, Garnsey JJ, Koenig E, Langston SP, Manfredi M, Narayanan U, Rolfe M, et al. MLN4924, a NEDD8-activating enzyme inhibitor, is active in diffuse large B-cell lymphoma models: rationale for treatment of NF-\{kappa\}B-dependent lymphoma. Blood. 2010; 116:1515-1523.

18. Soucy TA, Smith PG, Milhollen MA, Berger AJ, Gavin JM, Adhikari S, Brownell JE, Burke KE, Cardin DP, Critchley $\mathrm{S}$, Cullis CA, Doucette A, Garnsey JJ, et al. An inhibitor of NEDD8-activating enzyme as a new approach to treat cancer. Nature. 2009; 458:732-736.

19. Shah JJ, Jakubowiak AJ, O'Connor OA, Orlowski RZ, Harvey RD, Smith MR, Lebovic D, Diefenbach C, Kelly K, Hua Z, Berger AJ, Mulligan G, Faessel HM, et al. Phase I Study of the Novel Investigational NEDD8-Activating Enzyme Inhibitor Pevonedistat (MLN4924) in Patients with Relapsed/Refractory Multiple Myeloma or Lymphoma. Clin Cancer Res. 2016; 22:34-43.

20. Swords RT, Erba HP, DeAngelo DJ, Bixby DL, Altman JK, Maris M, Hua Z, Blakemore SJ, Faessel H, Sedarati F, Dezube BJ, Giles FJ, Medeiros BC. Pevonedistat (MLN4924), a First-in-Class NEDD8-activating enzyme inhibitor, in patients with acute myeloid leukaemia and myelodysplastic syndromes: a phase 1 study. British journal of haematology. 2015; 169:534-543.

21. Sheridan JP, Marsters SA, Pitti RM, Gurney A, Skubatch M, Baldwin D, Ramakrishnan L, Gray CL, Baker K, Wood WI, Goddard AD, Godowski P, Ashkenazi A. Control of TRAIL-induced apoptosis by a family of signaling and decoy receptors. Science. 1997; 277:818-821.

22. Vonarbourg C, Stolzenberg MC, Holzelova E, Fischer A, Deist FL, Rieux-Laucat F. Differential sensitivity of Jurkat and primary $\mathrm{T}$ cells to caspase-independent cell death triggered upon Fas stimulation. Eur J Immunol. 2002; $32: 2376-2384$.
23. Riboldi E, Daniele R, Cassatella MA, Sozzani S, Bosisio D. Engagement of BDCA-2 blocks TRAIL-mediated cytotoxic activity of plasmacytoid dendritic cells. Immunobiology. 2009; 214:868-876.

24. Tromp JM, Tonino SH, Elias JA, Jaspers A, Luijks DM, Kater AP, van Lier RA, van Oers MH, Eldering E. Dichotomy in NF-kappaB signaling and chemoresistance in immunoglobulin variable heavy-chain-mutated versus unmutated CLL cells upon CD40/TLR9 triggering. Oncogene. 2010; 29:5071-5082.

25. Chan H, Bartos DP, Owen-Schaub LB. Activationdependent transcriptional regulation of the human Fas promoter requires NF-kappaB p50-p65 recruitment. Molecular and cellular biology. 1999; 19:2098-2108.

26. Singh NP, Nagarkatti M, Nagarkatti PS. Role of dioxin response element and nuclear factor-kappaB motifs in 2,3,7,8-tetrachlorodibenzo-p-dioxin-mediated regulation of Fas and Fas ligand expression. Molecular pharmacology. 2007; 71:145-157.

27. Baetu TM, Kwon H, Sharma S, Grandvaux N, Hiscott J. Disruption of NF-kappaB signaling reveals a novel role for NF-kappaB in the regulation of TNF-related apoptosis-inducing ligand expression. J Immunol. 2001; 167:3164-3173.

28. Elgueta R, Benson MJ, de Vries VC, Wasiuk A, Guo Y, Noelle RJ. Molecular mechanism and function of CD40/ CD40L engagement in the immune system. Immunological reviews. 2009; 229:152-172.

29. Rickert RC, Jellusova J, Miletic AV. Signaling by the tumor necrosis factor receptor superfamily in B-cell biology and disease. Immunological reviews. 2011; 244:115-133.

30. You M, Ku PT, Hrdlickova R, Bose HR, Jr. ch-IAP1, a member of the inhibitor-of-apoptosis protein family, is a mediator of the antiapoptotic activity of the v-Rel oncoprotein. Molecular and cellular biology. 1997; 17:7328-7341.

31. Stehlik C, de Martin R, Kumabashiri I, Schmid JA, Binder BR, Lipp J. Nuclear factor (NF)-kappaB-regulated $\mathrm{X}$-chromosome-linked iap gene expression protects endothelial cells from tumor necrosis factor alpha-induced apoptosis. The Journal of experimental medicine. 1998; 188:211-216.

32. Neumann L, Pforr C, Beaudouin J, Pappa A, Fricker N, Krammer PH, Lavrik IN, Eils R. Dynamics within the CD95 death-inducing signaling complex decide life and death of cells. Mol Syst Biol. 2010; 6:352.

33. Safa AR. c-FLIP, a master anti-apoptotic regulator. Experimental oncology. 2012; 34:176-184.

34. MacFarlane M, Harper N, Snowden RT, Dyer MJ, Barnett GA, Pringle JH, Cohen GM. Mechanisms of resistance to TRAIL-induced apoptosis in primary B cell chronic lymphocytic leukaemia. Oncogene. 2002; 21:6809-6818.

35. Schug ZT, Gonzalvez F, Houtkooper RH, Vaz FM, Gottlieb E. BID is cleaved by caspase- 8 within a native complex on 
the mitochondrial membrane. Cell death and differentiation. 2011; 18:538-548.

36. Paiva C, Godbersen JC, Berger A, Brown JR, Danilov AV. Targeting neddylation induces DNA damage and checkpoint activation and sensitizes chronic lymphocytic leukemia B cells to alkylating agents. Cell death \& disease. 2015; 6:e1807.

37. Danilov AV, Danilova OV, Klein AK, Huber BT. Molecular pathogenesis of chronic lymphocytic leukemia. Curr Mol Med. 2006; 6:665-675.

38. Scarfo L, Ghia P. Reprogramming cell death: BCL2 family inhibition in hematological malignancies. Immunology letters. 2013; 155:36-39.

39. Davids MS, Letai A. Targeting the B-cell lymphoma/ leukemia 2 family in cancer. Journal of clinical oncology. 2012; 30:3127-3135.

40. Gerecitano JF, Roberts AW, Seymour JF, Wierda WG, Kahl BS, Pagel JM, Puvvada S, Kipps TJ, Anderson MA, Dunbar M, Zhu M, Gressick L, Wagner L, et al. (2015). A Phase 1 Study of Venetoclax (ABT-199 / GDC-0199) Monotherapy in Patients with Relapsed/Refractory NonHodgkin Lymphoma. American Society of Hematology Annual Meeting. (Orlando, FL: Blood).

41. Itchaki G, Brown JR. The potential of venetoclax (ABT199) in chronic lymphocytic leukemia. Ther Adv Hematol. 2016; 7:270-287.

42. Davis RE, Brown KD, Siebenlist U, Staudt LM. Constitutive nuclear factor kappaB activity is required for survival of activated B cell-like diffuse large B cell lymphoma cells. The Journal of experimental medicine. 2001; 194:1861-1874.

43. Snell V, Clodi K, Zhao S, Goodwin R, Thomas EK, Morris SW, Kadin ME, Cabanillas F, Andreeff M, Younes A. Activity of TNF-related apoptosis-inducing ligand (TRAIL) in haematological malignancies. British journal of haematology. 1997; 99:618-624.

44. Dicker F, Kater AP, Fukuda T, Kipps TJ. Fas-ligand (CD178) and TRAIL synergistically induce apoptosis of CD40-activated chronic lymphocytic leukemia B cells. Blood. 2005; 105:3193-3198.

45. Zonta F, Pagano MA, Trentin L, Tibaldi E, Frezzato F, Gattazzo C, Martini V, Trimarco V, Mazzorana M, Bordin L, Semenzato G, Brunati AM. Lyn-mediated procaspase 8 dimerization blocks apoptotic signaling in B-cell chronic lymphocytic leukemia. Blood. 2014; 123:875-883.

46. Travert M, Ame-Thomas P, Pangault C, Morizot A, Micheau O, Semana G, Lamy T, Fest T, Tarte K, Guillaudeux T. CD40 ligand protects from TRAIL-induced apoptosis in follicular lymphomas through NF-kappaB activation and up-regulation of c-FLIP and Bcl-xL. J Immunol. 2008; 181:1001-1011.
47. Vogler M, Butterworth M, Majid A, Walewska RJ, Sun XM, Dyer MJ, Cohen GM. Concurrent up-regulation of BCL-XL and BCL2A1 induces approximately 1000-fold resistance to ABT-737 in chronic lymphocytic leukemia. Blood. 2009; 113:4403-4413.

48. Soderquist R, Bates DJ, Danilov AV, Eastman A. Gossypol overcomes stroma-mediated resistance to the BCL2 inhibitor ABT-737 in chronic lymphocytic leukemia cells ex vivo. Leukemia. 2013; 27:2262-2264.

49. Ferreira KS, Kreutz C, Macnelly S, Neubert K, Haber A, Bogyo M, Timmer J, Borner C. Caspase-3 feeds back on caspase-8, Bid and XIAP in type I Fas signaling in primary mouse hepatocytes. Apoptosis : an international journal on programmed cell death. 2012; 17:503-515.

50. Inoue S, Harper N, Walewska R, Dyer MJ, Cohen GM. Enhanced Fas-associated death domain recruitment by histone deacetylase inhibitors is critical for the sensitization of chronic lymphocytic leukemia cells to TRAILinduced apoptosis. Molecular cancer therapeutics. 2009; 8:3088-3097.

51. Frenzel LP, Patz M, Pallasch CP, Brinker R, Claasen J, Schulz A, Hallek M, Kashkar H, Wendtner CM. Novel X-linked inhibitor of apoptosis inhibiting compound as sensitizer for TRAIL-mediated apoptosis in chronic lymphocytic leukaemia with poor prognosis. British journal of haematology. 2011; 152:191-200.

52. Loeder S, Zenz T, Schnaiter A, Mertens D, Winkler D, Dohner H, Debatin KM, Stilgenbauer S, Fulda S. A novel paradigm to trigger apoptosis in chronic lymphocytic leukemia. Cancer research. 2009; 69:8977-8986.

53. El-Mesery M, Seher A, Stuhmer T, Siegmund D, Wajant H. MLN4924 sensitizes monocytes and maturing dendritic cells for TNF-dependent and -independent necroptosis. Br J Pharmacol. 2015; 172:1222-1236.

54. Wolenski FS, Fisher CD, Sano T, Wyllie SD, Cicia LA, Gallacher MJ, Baker RA, Kirby PJ, Senn JJ. The NAE inhibitor pevonedistat (MLN4924) synergizes with TNF- $\alpha$ to activate apoptosis. Cell Death Discovery. 2015; 1:15034.

55. Danilov AV. Targeted therapy in chronic lymphocytic leukemia: past, present, and future. Clinical therapeutics. 2013; 35:1258-1270.

56. Zhao L, Yue P, Lonial S, Khuri FR, Sun SY. The NEDD8activating enzyme inhibitor, MLN4924, cooperates with TRAIL to augment apoptosis through facilitating c-FLIP degradation in head and neck cancer cells. Molecular cancer therapeutics. 2011; 10:2415-2425.

57. Neron S, Suck G, Ma XZ, Sakac D, Roy A, Katsman Y, Dussault N, Racine C, Branch DR. B cell proliferation following CD40 stimulation results in the expression and activation of Src protein tyrosine kinase. Int Immunol. 2006; 18:375-387. 
58. Humphries LA, Godbersen JC, Danilova OV, Kaur P, Christensen BC, Danilov AV. Pro-apoptotic TP53 homolog TAp63 is repressed via epigenetic silencing and B-cell receptor signalling in chronic lymphocytic leukaemia. British journal of haematology. 2013; 163:590-602.
59. Danilov AV, Neupane D, Nagaraja AS, Feofanova EV, Humphries LA, DiRenzo J, Korc M. DeltaNp63alphamediated induction of epidermal growth factor receptor promotes pancreatic cancer cell growth and chemoresistance. PloS one. 2011; 6:e26815. 